\title{
Loss of Control: Experiences of Depression in Thai Men
}

\section{Somporn RUNGREANGKULKIJ ${ }^{1, *}$, Ingkata KOTNARA ${ }^{1}$, Waraluk KITTIWATANAPAISAN ${ }^{1}$ and Suwanna ARUNPONGPAISAL ${ }^{2}$}

\author{
${ }^{1}$ Centre for Research and Training on Gender and Women's Health, Faculty of Nursing, \\ Khon Kaen University, Khon Kaen 40002, Thailand \\ ${ }^{2}$ Faculty of Medicine, Khon Kaen University, Khon Kaen 40002, Thailand
}

('Corresponding author's e-mail: somrun@kku.ac.th)

Received: 22 February 2017, Revised: 20 November 2017, Accepted: 21 December 2017

\begin{abstract}
In comparison to Thai women, Thai men do not seek care or treatment for depressive symptoms. The literature on men with depression reports that depressive symptoms are viewed as unmasculine. However, little is known about how Thai men experience depression. This qualitative study aimed to understand how Thai men understand having a depressive disorder. Twenty-one male patients were recruited from a psychiatric out-patient clinic and a university hospital in northeastern Thailand. The patients lived with major depressive disorder for at least one year. Data were collected by in-depth interviews and were tape-recorded. Data were transcribed verbatim and analyzed by content analysis. Six themes emerged which were related to masculinity: (a) a loss of control, (b) feeling upside-down, (c) personal weakness, (d) a lack of understanding, (e) social isolation, and (f) a wish to lean on something. The findings are discussed in the context of existing research on depression in men and within the context of masculinity. Recommendations for future research on male depression and implications for clinical practice with men are presented.
\end{abstract}

Keywords: Men, masculinity, depression, depressive disorder, Thai

\section{Introduction}

Depression is one of the leading causes of disability worldwide leading to suicide [1]. Over the last 30 years, globally, the rate of depression in men has been half of that in women [2]. Similarly, the Thai Department of Mental Health [3] reports that the prevalence of depressive disorders is higher in women than in men (2.9: 1.7). The suicide rate in Thai men, however, is 4 times higher than in women [4]. There is a possible explanation why depression prevalence is lower in men, based on the masculine gender role. First, men may deny depressed emotions, such as sadness or crying, because they goes against masculine identity. Second, men may not be aware of, or have difficulty in verbalizing, their depressive symptoms. Third, men may express depressive symptoms in different ways than women, such as hiding negative emotions by indulging in risk-taking and antisocial behaviors such as aggression, deliberate self-harm, or drug and alcohol abuse [5,6].

Frequently, men across cultures do not seek care or treatment for depressive symptoms because of how they conceptualize depression and because of concerns of losing their masculine image [2,7]. Gender roles are socially-constructed and, in many cultures, men are usually expected to be strong, self-reliant, in control, and be a leader and a financial provider for the family. When men cannot perform these expected family and societal roles, they may view themselves as being inadequate, which can add to the existing severity of depression. The onset of a mental illness can also enormously increase the pressure to maintain a strong male image [8]. Previous research has established that, with increasing symptoms and the inability to perform their usual activities, men may view themselves as being weak and have self- 
http://wjst.wu.ac.th

doubts about their masculinity [8]. Evidence from the literature review [9] consistently shows that men delay seeking health care and ignore their mental health problems. Besides their perception that depression is as a sign of failure, they also struggle with the perception of losing a sense of control and power, which causes additional vulnerability [7,8]. Men who experienced depression often refer to it as "stress" rather than "depression" [10].

Although previous studies have contributed to a better understanding of depression in men, there has been no qualitative study about the experiences of Thai men who have depression. Gender is socially constructed; therefore, Thai men may give different meaning to living with depression compared to other cultures. One qualitative study [11] has described perceptions of depression with elderly Thai immigrants. Key informants included 10 Thai immigrant men and 10 Thai immigrant women who had experienced depression or had friends or relatives who had experienced depression. The participants attended a Thai temple and lived in a metropolitan community in Illinois, USA. They found that both men and women described depression as feelings of disappointment and loneliness accompanied by obsessive thoughts of existing problems related to health, living situations, family members, and jobs. More men than women described depression as feeling of pressure in the mind due to their living situations and family relationships. Nevertheless, this study was conducted with Thai participants living in the US, with likely US cultural influences on their perceptions. In light of the very limited research on Thai men's experience of depression, this qualitative study was designed to explore the sense of meaning Thai men give to living with a major depressive disorder.

\section{Method}

This research is based on interview data drawn from an earlier exploratory descriptive study [12] to explain the perception of depression, symptom management, and the perception of treatments. Key informants included patients who had been diagnosed with major depressive disorder for at least one year. A total of 42 participants, including 21 men and 21 women, were purposively recruited from the outpatient departments of 2 hospitals; a psychiatric hospital and a psychiatric unit within a university teaching hospital, both in Northeastern Thailand. The inclusion criteria were patients aged between 18 59 years who were diagnosed with major depressive disorder by a psychiatrist. For this research paper, data were selected only from 21 male patients.

\section{Data collection}

Data were collected using an in-depth, semi-structured interview. The interviews consisted of 2 parts. In the first part, participants were asked about symptoms, symptom management, and their perception of the causes of illness and treatments. The second part, which is the focus of this paper, was the meaning of having depression. A question was asked at the end of the interviews, "what is it like to live with depression?" The interviews, which lasted from 45 to $90 \mathrm{~min}$, were audiotaped and transcribed verbatim by a professional transcription services. When participants tell their stories, they may experience discomfort or distress. They may get upset or stressed. If that was the case in the interviews, the researcher would stop the interview and provide the participants with some time to recuperate. When it appeared that the participants had recovered, the researcher checked to make sure that they were ready before going on with the interview. If the participants were still feeling distressed, the researcher would terminate the interview and refer the participants to their therapist.

\section{Data analysis}

Data on Thai men's meaning of living with depression were extracted from the 21 men's verbatim transcripts and analyzed using content analysis based on Colaizzi's 7 step method [13]; the first step focused on reading the interview transcriptions in order to understand the meaning as a whole, followed by the extraction of significant statements. The next step included the formulation of meanings from significant statements, which were then organized into themes. Themes were subsequently integrated into an exhaustive description. The $6^{\text {th }}$ step consisted of formulating the essential structure of the phenomenon. 
http://wjst.wu.ac.th

The main goal of content analysis through the course of this study was to derive understanding of the meaning of living with depression from Thai men's perspectives.

The credibility and trustworthiness of data analysis were assured by 3 processes. Firstly, data were collected by experienced qualitative researchers $(2$ psychiatric nurses who had a specialty in depression and qualitative research, and one adult nurse who had expertise in qualitative research). Secondly, researchers independently reviewed the transcripts to identify common themes. They then met and discussed the matter together to ensure the credibility of the findings and to reach a consensus on emerging themes. Finally, the researchers invited the participants to attend a group research finding presentation (member checking) [14]. However, only 7 male informants could participate in this confirmation process, as the remainder was unavailable due to their work schedules and transportation issues. After data analysis ended, the researchers found that the meaning of having depression was related to masculinity. After presenting research findings to the attending participants, the researchers asked the participants about "being a Thai man" and to comment on how "being a man" influenced their experiences of depression. Therefore, reference to masculinity was confirmed by the attending participants. Those attending agreed with the findings and offered further clarification and explanation of the researchers' interpretations.

\section{Ethical review}

The study was reviewed and approved by the Human Ethics Committee of Khon Kaen University (HE\#531043).

\section{Findings}

\section{Participant demographics}

The mean age of the participants was 43.6 years (range 18 to 58 years). The majority (66\%) were married (14 of 21) and had a completed a bachelor's degree or higher (11 of 21). Fifteen participants were employed: occupations included laborer, government employee, and business owner. Three were college students. The other 3 were retirees with income from pensions. The average duration of their depression was 7.8 years (range 1 to 35 years). All were being treated with antidepressants.

\section{Qualitative findings}

Participants expressed an overall meaning of depression as a condition of loss of control, especially in thought. Masculinity identity was considered to have an impact on the participants' depression. Six themes emerged during the analysis of the interview transcripts, as below: (a) loss of control, (b) feeling upside-down, (c) personal weakness, (d) a lack of understanding, (e) social isolation, and (f) a wish to lean on something.

\section{Theme 1: Loss of control}

Loss of control means that a participant expressed the loss of his ability to control his own thoughts. In Thai society, the image of men is generally of a strong man, a leader, self-reliant, in control, successful, and a protector. For men with depression, they feel that they cannot solve problems on their own. They feel trapped within their own thoughts. They have obsessive thoughts of existing problems. They feel they suffer from a lack of ability to be in control. All participants in this study expressed suffering from the inability to control their thoughts.

A 58 year old participant stated that: "There is no cure for this illness. It seems like it keeps building up. The illness is controlling what I think. I don't want to think but I can't stop. I keep thinking. This is crazy. I don't want to think but the thoughts keep coming up to the surface. When I am alone, I think even more. There is no cure! I will die with it." (P1)

Another participant, a 20 years old student also expressed being trapped within the thoughts: "the thoughts just came. They just came out. Sometimes I don't want to think but the thoughts just came. I try to stop them, but I can't." (P11) 
http://wjst.wu.ac.th

\title{
Theme 2: Feeling upside-down
}

In this study, many men mentioning that one of the symptoms of depression was when your ability to do things is low. They found that their lives changed from having self-reliance and self-confidence and hope for the future, to having a hopeless life. They felt the more they tried to get out, the deeper they went toward the bottom. They could not stand up on their own. They had self-doubt in their ability to do things.

"I felt like my life was changed upside-down. I was motivated and energetic about the future. But at one point, I had become still and then driven down. I felt like nothing was important. I wanted to get away. I kept dwelling on many thoughts. I couldn't sleep. I worried about many things. I wondered why there was no way out of this. I thought I would just be stuck down here. My soul was driven down. I wanted to just give up. The problems kept pushing me down; my back against the wall. I tried to find the way out but I couldn't. I kept thinking and my head hurt. I had to live with the pain. When I experienced all these symptoms, I felt like I fell off the chart." (P3)

A 54 year old participant described his experience of depression like "being caught in a storm”. He felt like his life had turned upside-down. He described how his life was spiraling down to the point that he felt trapped: “It's like being caught in a storm, the storm is very strong, I feel like I'm going upside-down. I have tried to solve it, manage it, but it was the same. I couldn't slow it down or manage it successfully, I was brought down so low, I couldn't solve the problem, it came right back at me, there were many questions that came up and I was stuck. I tried to think but I couldn't come up with any answers, it went around and around." (P4)

Another participant aged 55 said "I had never been so disappointed, I felt like something went through me. I felt like I flipped upside-down and fell down hard. I had many questions going around and started asking questions. I questioned myself. I questioned if I could step forward. I got very confused." (P18)

Theme 3: Personal weakness

The participants talked about themselves being weak. Personal weakness means the loss of strength, self-confidence, and the ability to problem-solve successfully. They said that it was the feeling of being weak which is the opposite of masculine identity. The masculine image of being a man is being strong with self-confidence. When the participants identified themselves as being weak, they have a hard time dealing with the feeling. The men tried to conceal "the personal weakness". They also tried to get over the weakness and felt worse when they could not. When men suffer from depression, they feel like a failure. Participants labeled depression as a sign of weakness. A 53-year-old early-retired participant said "Depression changes a person from being ambitious and proud to nothing. One's self confidence is low or gone...How should I say this? I think the weakness within me has come out. I don't have any selfconfidence. I think...my body strength is lower and so is my libido. That's what I believe. It shows weakness. I think for men, we have pride. Men have to be strong and cannot show weakness to others." (P10)

\begin{abstract}
"It's hard to accept that I have depression. Why? Because it's weak. I don't want to accept that I am weak and that I can't handle it. But I couldn't keep it inside any longer. I lost all confidence. I have tried my hardest not to be weak, to gain energy to fight. But it takes a lot of the internal energy to hide the depression, to appear normal. I think for men, we have pride. We want to be strong. We don't want to reveal that we are weak." (P17)
\end{abstract}

Theme 4: Lack of understanding

The participants expressed feeling like nobody understands them. The lack of understanding went both ways. The participants did not know how to communicate the internal changes to others, while feeling like others did not understand them. All participants said that depression was a condition that was hard for people to understand because the symptoms and the suffering were not obvious, like physical 
http://wjst.wu.ac.th

illnesses. For instance, a participant, who is a student, wanted to tell his parents. However, he didn't know how to communicate and explain how he felt and the changes within him. When he was trying to talk to the parents, the parents denied him due to their lack of understanding. "I can tell you that only a depressed person understands and knows that they are sick. It is difficult to explain to others. Some people said you don't look sick. Outsiders, they don't know. They don't understand. But I've noticed myself that I have changed so I told my parents. At first, they just laughed at me. They said "oh! You're just tired. You must have a fever.' I told them I didn't have a fever. I told them my body is normal but I just didn't feel right. I told them I didn't have any motivation or energy. My parents still didn't understand. My parents said 'it's in your head. You must have imagined things. Just take some vitamins for your brain." (P11)

Because of the masculine image of self-reliance, many participants had obsessive thoughts of their existing problems. They tried to solve the problems themselves. They did not seek help from others. They did not tell their closest friends or loved ones. This created a lack of understanding. When the loved ones did not understand, they tried to encourage the participants to be strong, powerful, and successful. This lack of understanding, and the use of words of encouragement to keep the masculine identity intact, makes men with depression frustrated. They feel the lack of understanding. Many times, the participants expressed that they wanted to feel better, but the depression prevented them from getting up on their own.

A 54-year-old man said: "It's rare that other people will accept you. Even my own wife; as an example, I told her that I didn't feel like working. I wanted to take a day off. I didn't want to face anybody that day. She didn't support me. I wondered why she didn't help me. I truly didn't feel like talking to anybody. I wanted to take off. I felt like my wife was pushing me toward things that I didn't want to face. She felt like she should push me toward success. But for me, it wasn't. I wondered why she did not ask me how I felt. She should have suggested that I rest or how I could feel better. It's hard to find someone who can understand you." (P7)

When people around them showed lack of understanding, the depressed men suffered by themselves. They felt frustrated and suffered from feeling like there was no exit to their problems. This could put them at risk for suicide. As an example, a 52-year-old participant said that, before he had experienced depression, he didn't understand how a person could hurt himself. But when it happened to him, he understood why people attempted suicide. He described it as the following:

"When there is no way out, suicide is a possibility. At first, I was against harming myself or thinking about suicide. But now I understand people who've committed suicide. I understand that they must have suffered. The suffering must be so severe. This type of suffering, if you have never experienced or studied it, it is probably hard to understand." (P21)

\section{Theme 5: Social isolation}

Social isolation is social withdrawal and a change in doing activities or in the social interaction of the participants. The participants change from enjoying meeting people and doing activities to becoming isolated. They do not feel like doing things that they used to enjoy. Due to their lack of self-confidence and their obsessive thoughts, many men chose to stay isolated. They found it hard to go out and to act according to society's expectations. They found it took too much energy to live up to masculine identity.

A 48-year-old man revealed that he used to be social; however, he had changed since he had depression. When he had to socialize, he concealed his low mood and tried to act normally, as follows.

"Normally, I was an outgoing person. I liked to have fun and to go out with friends. I was not a shy person. But now I am very isolated, because I don't feel self-confident. I can't think so clearly. I don't have much energy. Before I could get along and had fun with anybody. But now I don't want to go out with anybody. I just want to be by myself. The depression really affects my life. I look like 
http://wjst.wu.ac.th

a normal person but, deep down, I don't feel normal. I might be smiling on the outside, but I actually cry on the inside. I feel very sad in my heart. My smile is from the expectation, but not really truly from happiness." (P15)

A 45-year-old male teacher shared his experience of feeling withdrawn from society, as follows:

"The bad part is the expectation from society. They expect you to participate. When I was normal, I would normally go out with friends and have fun. But when I am depressed like this, I only want to be by myself. I want to be quiet. I don't want to go out. When I get any party invitation, I ask my wife or daughter to go instead. It's been over a year that I really don't want to go out." (P16)

\section{Theme 6: Wish to lean on something}

Wishing to lean on something was a common feeling that the participants expressed. When they felt that they were very deep down in their own problems, they suffered from their inability to find their way out. They wished to lean on someone or find some way to feel better. Many times, the men turned to drugs and alcohol. They did not have someone who could understand them. They did not know how to express themselves. Eventually, when the men sought professional help and were diagnosed with depression, they were able to lean on the nurses. They had someone to talk to. They had learnt that they could talk to the doctors and nurses about their problems. A 58-year-old early-retired man said, "Depression is the condition that makes you want to lean on something. It is a condition where you can't rely on yourself. I've tried to make other people understand me. That was kind of leaning on something too. Some people rely on drugs or alcohol and end up dependent. Alcohol is like medicine. When I drink, I feel less anxious and worried. It helps me forget my problems. But if I keep drinking, it will become a habit. I feel restless. After a while, I would end up addicted to alcohol. If I can't shake off this habit, it'll become worse. I will go deeper and further down. Eventually, I won't be able to find my way out." (P19)

When the men felt severely depressed to the point of having to get professional help, they found talking to professionals helped them find the exit out of the problems. Their attitude of having to hide their problems was changed to accepting and revealing their problems and feelings to the professionals. This attitude is different from the masculine image. The men felt the need to be listened to and to rely on someone.

"In the past, I felt like I had to carry all the responsibilities. I am a man. I have to be a leader of the family. I have to take care of all the problems. I couldn't talk to anybody about the troubles deep inside me. When I was very sick, I had a chance to talk to the doctors and nurses, I felt better. I had the opportunity to talk and to be listened to. I had a chance to talk to someone to find the way out. This illness, when you can talk, you feel better. The symptoms will subside." (P12)

The above 6 interpretations of depression having an impact on manhood were confirmed when the researchers presented their interpretations of the research findings to key informants.

For men's thinking, we have to be strong, confident, and a leader for the children and family. We have to lead them to the goals. We have to be strong and be able to handle hardships more than women. But when you have depression, you just fail to think these ways. You just give up. (P17)

In the past, I had confidence that I had to be a leader. But after I had the depression, I've lost all my confidence. My wife was the follower but now she has to step up. Sometimes, I have to let her lead me because she has better ideas and her brain works better than mine. When I have lost my self-confidence, it has affected people around me. My children, who used to be respectful, start questioning why you are like this? You have never been like this! That leads to the poor attitude and relationship between father and children. I don't dare to explain to them why things work this way or that way. I lost my confidence, even with my children." (P 12) 


\section{Discussion}

This research contributes to new knowledge on depression in Thai society in explaining depression based on social construction, especially related to masculinity. The results shed some light on the knowledge of depression beyond the individual level. This is the first research finding that provides insight into Thai men's experiences with depression. The Thai men's experiences are found to be similar to depressed men in other countries. Many studies about men's depression find that depressed men try to maintain masculine role norms. Even when they know that they do not feel right, they try to solve the problems on their own. They have to conceal their feelings. They feel trapped and lose control of themselves. The masculine role norms prevent the men with depression from seeking help. They do not talk about their problems, so the people around them do not understand what they are going through. These findings are in line with previous research that supports the notion that depression impacts the sense of masculine identity in similar ways as are found in other cultures.

\section{Loss of control}

The findings of this study supported the notion of rumination being related to depression [15]. Engaging in rumination was mentioned by all participants. Thai male identity includes the sense of being strong, tough, self-reliant, competent, and successful. They tried to solve problems by themselves but were unsuccessful. The more they tried, the more they suffered from rumination. The unresolved ruminations influenced their sense of loss of self-control, energy, self-confidence, and hope. This lack of control affected their masculine identity. Like in many other cultures, Nolen-Hoeksema [15] found that women respond to stress with more rumination than men. However, women perceive rumination as thinking a lot about unresolved relationship problems. No women mentioned it in terms of loss of control, while men in this study mentioned loss of control as a sequence of rumination. This may because women are not expected to be self-reliant from social norms.

\section{Feeling upside-down}

The men in this study said that the depression had changed their lives. Thai society expects men to be leaders and successful. However, when they experience depression, they feel hopeless, worthless, and lack confidence. The participants had changed from looking into the future and leading themselves and their families to success to only looking into themselves and being stuck with their problems. They felt life was going down, not up as before. These meanings were similar to depressed men in England who described their experiences as dark, tormented, and imprisoned [2]. Young Afro-American men with depression also described their experience as "feeling beat down" or "head down" [16]. Elderly Thai men who lived in the United States mentioned experience of depression as "sinking feeling" [11].

\section{Personal weakness}

Thai men's identity includes being strong, successful, self-confidence, self-reliant, and a protector. When they feel stuck in their problems and unable to solve them, they have an additional pressure to push them down. The feelings they have experienced are the opposite of being a man. This is different than in women with depression. In Thai society, women are expected to be weak and to be dependent. When men cannot reach social expectations, they consequently can view themselves as weak. Their first reaction is to try to conceal their feelings. They try to act normally and perform their usual activities in order to be a man [17]. Feeling weakness has been found in many men with depression in previous studies $[16,18]$.

\section{Lack of understanding}

Most men did not express typical depression symptoms such as feeling sad or crying; however, this could be hidden in their thoughts or reduced energy or interest in things around them. Therefore, it can be hard for others to understand when men experience depression. This could make a depressed person's life more difficult, as people around him try to push him to maintain his former energetic roles. For example, in this study, one man stated how much his wife tried to push him up in order to bring him back to 
http://wjst.wu.ac.th

normal. It could add frustration to an already depressed man if people around him expect him to continue in traditional masculine roles during depression.

\section{Social isolation}

Social isolation is a common symptom found in both men and women with depression [19]. The men in this study compared social isolation with masculine role norms. When they did not feel selfconfident, they did not want to go out. They felt like they were not a real man. On the other hand, women with depression talk about social isolation because they feel alone [11]. The same reason for social isolation in men is also mentioned in other studies $[2,16,20]$ : the attempt to conceal depressive symptoms [17-20]. Social isolation also results in men limiting their opportunity to get help [20].

\section{Wish to lean on something}

Some participants reported suffering from being wrapped up with their own thoughts and having no solution to their problems. They wished that they could lean on someone. They wished someone could understand them. However, many participants hesitated to get help because of the desire to maintain a masculine image. Some men avoided being perceived as weak; consequently, they decided to lean on alcohol. Alcohol dependence is quite prevalent in Thai men. In some studies, alcohol misuse and suicide were found to be related to male depression $[6,17]$. This is also true in the experiences of Thai men. They turn to alcohol, hoping that it will help them feel better. Drinking is socially acceptable for Thai men because it is stereotypical of Thai manhood. Therefore, when men decide to lean on alcohol instead of professional help, they increase their risk of becoming alcohol dependent. In Thailand, the prevalence of alcohol misuse and suicide are higher in men than in women [4].

Participants reported that talking to therapists helped validate their internal distress. The depressed men had the need to be heard, accepted, and supported. A similar finding was also mentioned in Emslie et al. study [2,18]. Depressed men expressed that having significant others, such as a spouse, a partner, or close friends, could reduce their negative emotions [18]. The men in this study said that talking to professionals was helpful. When professionals commented to the men that depression makes them weak, not that they are weak, this rephrase made the participants feel better and more hopeful. They hoped that, when the depression is treated, the weakness goes away. This approach made it easier for the participants to accept help from professionals and to feel like they could maintain their masculinity. The approach made the men feel they are understood, and that their feelings were validated. The men found that they were able to increase their masculinity, even when they sought help from professionals. They had a sense of taking charge of the illness, because they sought help on their own [21]. Men with depression can increase their masculinity by rephrasing seeking help as a way in which depressed men show their courage. The help-seeking behavior is then perceived as showing leadership and taking risks, which suits the cultural norms and expectations of masculinity. When they express their needs, they show other men that they have effective ways of managing their negative emotions [2].

\section{Strengths and limitations}

This is the first Thai paper to describe the meaning Thai men give to living with depression. It provides information for mental health professionals and others to understand men's experiences. It is a space to open up discussion and greater understanding of Thai men's experiences with depression. Although a small sample, all participants who attended the research feedback session confirmed the researchers' interpretation of their data. Data for this research is part of a larger exploratory descriptive study, "The Perceptions of Depression of Thai Patients with Major Depressive Disorder using the Explanatory Model", and further data from that may provide fuller insights and confirmation of our findings. In addition, most participants were men in the workforce; therefore, their meanings may differ from other men's groups. 


\section{Conclusion and suggestions}

The findings indicated that masculine norms play a role in Thai men. Thai men suffer from ruminative thoughts about failing to carry on the traditional masculine roles. They experience a sense of loss of self-control, lack of understanding, a perception of personal weakness, and social isolation. The way the participants expressed changes due to depression were in terms of rumination and social isolation. This qualitative study provides new information on men's depression to inform future research and clinical practice. Firstly, masculinity is socially constructed; therefore, experiences of men living with depression need more study in diverse contexts, such as age, socioeconomic status, and sexuality. Secondly, there is an urgent need to conduct more research on how Thai men express depressive symptoms and recovery in order to provide gender-specific care. Thirdly, research on how masculinity affects help-seeking behaviors will yield information in order to develop appropriate programs for early treatment intervention. Finally, development of a male depression risk scale may be useful for screening depression in males.

Findings from this study provide a challenge for mental health professionals on how to integrate masculine identity as a risk factor in developing programs for depression prevention. As Thai men with depression suffer from ruminative thoughts, cognitive behavior therapy analyzing masculine core beliefs may be of benefit for Thai men. Educating families about how men with depression suffer from losing masculinity may help better understanding for ways to support men with depression, by moderating expectations and pressures to maintain masculinity norms during depressive episodes. Some men in this study were willing to share their problems with health care providers; therefore, psychiatrists or psychiatric nurses should not be biased in thinking that men will not express problems when they seek help from health care services. In addition, healthcare providers should affirm to men when they access help care that they are making a good decision.

\section{Acknowledgements}

We would like to thank all the men who gave up their time to take part in this study, and the Faculty of Nursing, Khon Kaen University, Thailand, for providing the funding for this study.

\section{References}

[1] World Health Organization. Depression. Available at: http://www.who.int/mediacentre/factsheets/ fs369/en, accessed July 2016.

[2] C Emsile, D Ridge, S Ziebland and K Hunt. Men's accounts of depression: Reconstructing or resisting hegemonic masculinity? Soc. Sci. Med. 2006; 62, 2246-57.

[3] Department of Mental Health, Ministry of Public Health. Report on Situations and Trends of Mental Health Managing for Developing Strategic Plan of the Eleventh National Economic and Social Development Plan 2012-2016. The Department of Mental Health, Ministry of Public Health, Bangkok, Thailand, 2008.

[4] Department of Mental Health, Ministry of Public Health. National Data Suicide Rate of Thailand. Available at: http://www.dmh.go.th/report/suicide/english, accessed July 2016.

[5] S Brownhill, K Wilhelm, L Barclay and V Schmied. Big build: Hidden depression in men. Aust. NZ J. Psychiat. 2005; 39, 921-31.

[6] EM Addis. Gender and depression in men. Clin. Psychol. Sci. Pract. 2008; 15, 153-68.

[7] JL Oliffe and MJ Phillips. Men, depression and masculinities: A review and recommendations. $J$. Mens Health 2008; 5, 194-202.

[8] WH Courtenay. Constructions of masculinity and their influence on men's well-being: A theory of gender and health. Soc. Sci. Med. 2000; 50, 1385-401.

[9] MP Galdas, F Cheater and P Marshall. Men and health help-seeking behaviour: Literature review. $J$. Adv. Nurs. 2005; 49, 616-23.

[10] R O'Brien, K Hunt and G Hart. It's caveman stuff, but that is to a certain extent how guys still operate: Men's accounts of masculinity and help seeking. Soc. Sci. Med. 2005; 61, 503-16. 
http://wjst.wu.ac.th

[11] R Soonthornchaiya R. and B Dancy. Perceptions of depression among elderly Thai immigrants. Ment. Health Nurs. 2006; 27, 681-98.

[12] S Rungreangkulkij, I Kotnara, W Kittiwatanapaisan and S Arunpongpaisal. Explanatory model based on perspectives of patients with depressive disorder (in Thai). J. Psychiatr. Assoc. Thailand 2013; 58, 29-40.

[13] P Colaizzi. Psychological Research as the Phenomenologist Views It. In: R Valle and M King (Eds.). Existential Phenomenological Alternatives for Psychology. Oxford University Press, New York, 1978, p. 48-71.

[14] HS Speziale. Designing Data Generation and Management Strategies. In: HS Speziale and DR Carpenter (Eds.). Qualitative Research in Nursing. $3^{\text {rd }}$ ed. Lippincott Williams \& Wilkins, Philadelphia, 2003, p. 27-42.

[15] S Nolen-Hoeksema. Gender differences in depression. Curr. Dir. Psychol. Sci. 2001; 10, $173-6$.

[16] D Perkins, P Kelly and S Lasiter. Our depression is different: Experiences and perceptions of depression in young black men with a history of incarceration. Arch. Psychiat. Nurs. 2014; 28, 167 73.

[17] EC Apesoa-Varano, JC Barker and L Hinton. Shards of sorrow: Older men's accounts of their depression experience. Soc. Sci. Med. 2015; 124, 1-8.

[18] C Chuick, J Greenfeld, S Greenberg, S Shepard, S Cochran and J Haley. A qualitative investigation of depression in men. Psychol. Men Masculin. 2009; 10, 302-13.

[19] JM Girard, JF Cohn, MH Mahoor, SM Mavadati, Z Hammal and DP Rosenwald. Nonverbal social withdrawal in depression: Evidence from manual and automatic analysis. Image Vis. Comput. 2014; 32, 641-7.

[20] LJ Ramirez and AT Badger. Men navigating inward and outward through depression. Arch. Psychiat. Nurs. 2014; 28, 21-8.

[21] JL Johnson, JL Oliffe, MT Kelly, P Galdas and JS Ogrodniczuk. Men's discourses of help-seeking in the context of depression. Sociol. Health Illn. 2012; 34, 345-61. 